DOI: https://doi.org/10.24297/jam.v20i.9023

\title{
Metallic Ratios : Beyond the Golden Ratio The Mathematical Relationships between different Metallic Means
}

\author{
Dr. Chetansing Rajput \\ M.B.B.S. (Mumbai University) India, Asst. Commissioner (Govt. of Maharashtra) \\ Email: chetansingkrajput@gmail.com
}

Lecture Link: https://www.youtube.com/watch?v=raosniXwRhw

Website: https://goldenratiorajput.com/

\begin{abstract}
This paper introduces the precise mathematical relationships between different Metallic Ratios. Certain distinctive mathematical correlations are found to exist between various Metallic Means. This work presents the explicit formulae those provide the exact mathematical relationships between different Metallic Ratios.
\end{abstract}

Keywords: Metallic Mean, Golden Ratio, Fibonacci sequence, Pi, Phi, Pythagoras Theorem, Divine Proportion, Silver Ratio, Golden Mean, Right Triangle, Pell Numbers, Lucas Numbers, Golden Proportion, Metallic Ratio

\section{Introduction}

The prime objective of this work is to introduce and elaborate the precise mathematical relationships between different Metallic Means.

Each Metallic Mean $\boldsymbol{\delta}_{\mathrm{n}}$ is the root of the simple Quadratic Equation $\mathbf{X}^{\mathbf{2}}-\mathbf{n X}-\mathbf{1}=\mathbf{0}$, where $\mathbf{n}$ is any positive natural number. Thus, the fractional expression of the $n^{\text {th }}$ Metallic Ratio is $\delta_{n}=\frac{n+\sqrt{n^{2}+4}}{2}$ Moreover, each Metallic Ratio can be expressed as the continued fraction:

$$
\boldsymbol{\delta}_{\mathbf{n}}=\mathbf{n}+\frac{\mathbf{1}}{\mathbf{n}+\frac{\mathbf{1}}{\mathbf{n}+\frac{\mathbf{1}}{\mathbf{n}+\ldots}}} ; \text { And hence, } \boldsymbol{\delta}_{\mathbf{n}}=\mathbf{n}+\frac{\mathbf{1}}{\boldsymbol{\delta} \mathbf{n}}
$$

More importantly, each Metallic Ratio can be expressed with a special Right Angled Triangle. Any $\mathrm{n}^{\text {th }}$ Metallic Mean can be accurately represented by the Right Triangle having its catheti $\mathbf{1}$ and $\frac{\mathbf{2}}{\mathbf{n}}$. Hence, the right triangle with one of its catheti $=\mathbf{1}$ may substantiate any Metallic Mean, having its second cathetus $=\frac{\mathbf{2}}{\mathbf{n}}$, where $\mathrm{n}=1$ for Golden Ratio, $\mathrm{n}=2$ for Silver Ratio, $\mathrm{n}=3$ for Bronze Ratio, and so on [1]. 
Such Right Triangle provides the precise value of $\mathrm{n}^{\text {th }}$ Metallic Mean by the generalised formula:

The $\mathrm{n}^{\text {th }}$ Metallic Mean $\boldsymbol{\delta}_{\mathrm{n}}=\frac{\text { Cathetus } 1+\text { Hypotenuse }}{\text { Second Cathetus }}=\frac{1+\text { Hypotenuse }}{2 / \mathrm{n}}$

Hence, the 1:2: $\sqrt{5}$ Triangle provides the Fractional Expression of Golden Ratio $(\boldsymbol{\varphi})=\frac{\mathbf{1}+\sqrt{\mathbf{5}}}{\mathbf{2}}$

Similarly, the right triangle $1: 1: \sqrt{2}$ represents the Silver Ratio $\boldsymbol{\delta}_{\mathbf{2}}=\frac{1+\sqrt{2}}{1}=2.41421356 \ldots$.

Likewise, the right triangle with its catheti $\mathbf{1}$ and $\frac{2}{3}$ provides the geometric substantiation of the Bronze Ratio, and so on [1].

Hence, such right angled triangle, with its catheti $\mathbf{1}$ and $\frac{\mathbf{2}}{\mathbf{n}}$; that provides the precisee fractional expression of the $\mathbf{n}^{\text {th }}$ Metallic Mean, is the quintessential form of that particular Metallic Mean $\left(\boldsymbol{\delta}_{\mathbf{n}}\right)$. Remarkably, every geometric feature of such "Fractional Expression Triangle" is the purest expression of $\boldsymbol{\delta}_{\mathbf{n}}$. Several intriguing geometric features of such "Fractional Expression Triangle" have been described in detail, in the works mentioned in References [1] and [2].

However, the main aim of present work is to introduce the accurate mathematical relationships between various Metallic Ratios. Certain couples of Metallic Ratios are found to reveal the accurate values of other Metallic Means, by particular mathematical formulae. Also, the trigonometric ratios of different Odd Powers of any Metallic Mean are observed to render the precise values of several other Metallic Ratios. The objective of this paper is to put forward such empirical formulae those provide such peculiar relationships between different Metallic Means.

\section{A Couple of Metallic Ratios generating another Metallic Mean:}

If $\mathbf{K}, \mathbf{m}$ and $\mathbf{n}$ are positive integers such that $\frac{\mathbf{m n}+\mathbf{4}}{\mathbf{m}-\mathbf{n}}=\mathbf{K}$

then, it is observed that

$\boldsymbol{\delta}_{\mathrm{k}}=\frac{\boldsymbol{\delta}_{\mathrm{m}} \times \boldsymbol{\delta}_{\mathrm{n}}+\mathbf{1}}{\boldsymbol{\delta}_{\mathrm{m}}-\boldsymbol{\delta}_{\mathrm{n}}}$ where $\boldsymbol{\delta}_{\mathrm{k},}, \boldsymbol{\delta}_{\mathrm{m}}$ and $\boldsymbol{\delta}_{\mathrm{n}}$ are the $\mathrm{k}^{\text {th }}, \mathrm{m}^{\text {th }}$ and $\mathrm{n}^{\text {th }}$ Metallic Means respectively.

For example, consider the $\mathbf{6}^{\text {th }}$ Metallic Ratio $\boldsymbol{\delta}_{6}=6.162277660168 \ldots .$.

And for instance, as the integers 6, 11 and 14 satisfy the condition: $\frac{11 \times 6+4}{11-6}=14$, hence the $6^{\text {th }}$ and the $11^{\text {th }}$ Metallic Means can give the precise value of $14^{\text {th }}$ metallic mean, by the abovementioned formula;

$$
\frac{\boldsymbol{\delta}_{11} \times \boldsymbol{\delta}_{6}+\mathbf{1}}{\boldsymbol{\delta}_{11}-\boldsymbol{\delta}_{6}}=\boldsymbol{\delta}_{14}=14.07106 \ldots \ldots
$$


Similarly, this $6^{\text {th }}$ Metallic Ratio $\left(\boldsymbol{\delta}_{6}\right)$ can also give the precise values of $7^{\text {th }}, 8^{\text {th }}, 10^{\text {th }}, 11^{\text {th }}, 14^{\text {th }}, 16^{\text {th }}, 26^{\text {th }}$ and $46^{\text {th }}$ Metallic Means, by this simple formula: $\boldsymbol{\delta}_{\mathrm{k}}=\frac{\boldsymbol{\delta}_{\mathrm{m}} \boldsymbol{\delta}_{\mathrm{n}}+\mathbf{1}}{\boldsymbol{\delta}_{\mathrm{m}}-\boldsymbol{\delta}_{\mathrm{n}}}$

Hence, if $\mathbf{n}=\mathbf{6}$

\begin{tabular}{|l|l|l|l|l|l|l|l|l|}
\hline $\mathbf{m}=$ & 7 & 8 & 10 & 11 & 14 & 16 & 26 & 46 \\
\hline $\mathbf{k}=$ & 46 & 26 & 16 & 14 & 11 & 10 & 8 & 7 \\
\hline
\end{tabular}

Moreover, the $6^{\text {th }}$ Metallic Ratio $\left(\boldsymbol{\delta}_{6}\right)$ also renders the precise values of various Metallic Means, with the Metallic Ratios smaller than itself; for example, as $\frac{\mathbf{6} \times \mathbf{5}+\mathbf{4}}{\mathbf{6 - 5}}=34$, hence the $\mathbf{6}^{\text {th }}$ and the $\mathbf{5}^{\text {th }}$ Metallic Means can give the precise value of $\mathbf{3 4 ^ { \text { th } }}$ Metallic Mean, by the same formula:

$\frac{\boldsymbol{\delta}_{6} \times \boldsymbol{\delta}_{5}+\mathbf{1}}{\boldsymbol{\delta}_{6}-\boldsymbol{\delta}_{5}}=\boldsymbol{\delta}_{34}=34.02938636592 \ldots \ldots$

Hence, if $\mathbf{m}=6$

\begin{tabular}{|l|l|l|l|l|}
\hline $\mathbf{n}=$ & 5 & 4 & 2 & 1 \\
\hline $\mathbf{k}=$ & 34 & 14 & 4 & 2 \\
\hline
\end{tabular}

And, just like the digit $\mathbf{6}$ exemplified here, any such integer can similarly generate the precise values of several Metallic Ratios; with this intriguing formula $\frac{\boldsymbol{\delta}_{\mathbf{m}} \boldsymbol{\delta}_{\mathbf{n}}+\mathbf{1}}{\boldsymbol{\delta}_{\mathrm{m}}-\boldsymbol{\delta}_{\mathbf{n}}}=\boldsymbol{\delta}_{\mathrm{k}}$ which is the Generalised Formula for any positive integers $\mathbf{k}, \mathbf{m}$ and $\mathbf{n}$ those satisfy the only prerequisite: $\frac{m n+4}{m-n}=k$

\section{Odd Powers of a Metallic Ratio:}

Different "Odd Powers" of a Metallic Ratio are also observed to render the precise values of various Metallic Means by the following formulae;

Consider $\boldsymbol{\delta}_{\mathbf{m}}$ as any $\mathbf{m}^{\text {th }}$ Metallic Ratio.

And let $G_{1}, G_{2}, G_{3} \ldots \ldots . .$. be the Integer Sequence associated with this Metallic Mean $\boldsymbol{\delta}_{\mathbf{m}}$, for example: Fibonacci Sequence for Golden Ratio $\left(\boldsymbol{\delta}_{\mathbf{1}}\right)$, Pell Sequence for Silver Ratio $\left(\boldsymbol{\delta}_{\mathbf{2}}\right)$, and so on. 
And, let $L_{1}, L_{2}, L_{3}$ be the Lucas Sequence associated with this Metallic Mean $\boldsymbol{\delta}_{\mathbf{m}}$, for example: the Lucas Sequence 1, 3, 4, 7, 11...... for Golen Ratio $\left(\boldsymbol{\delta}_{\mathbf{1}}\right)$, and the Pell-Lucas Sequence 2, 6, 14, 34, 82...... for Silver Ratio $\left(\boldsymbol{\delta}_{2}\right)$, and so on.

Let $\mathbf{n}$ be an Odd Positive Integer. The "Odd Powers" of a Metallic Ratio $\boldsymbol{\delta}_{\mathbf{m}}$ can give the precise values of various Metallic Means by the following three formulae;

Formula 1: $\quad \arctan \left(\boldsymbol{\delta}_{\mathbf{m}}\right)^{\mathbf{n}}+\arctan \left(\boldsymbol{\delta}_{\mathbf{m}}\right)^{\mathbf{n}+\mathbf{2}}=2 \times \arctan \left(\boldsymbol{\delta}_{\mathbf{k}}\right) ;$ where $\mathbf{k}=\mathbf{2} \times \mathbf{G}_{(\mathbf{n}+\mathbf{1})}$

Formula 2: $\quad \arctan (\boldsymbol{\varphi})^{\mathbf{n}}+\arctan (\boldsymbol{\varphi})^{\mathbf{n}+\mathbf{6}}=2 \times \arctan \left(\boldsymbol{\delta}_{\mathbf{k}}\right) ;$ where $\mathbf{k}=\mathbf{F}_{(\mathbf{n}+\mathbf{3})}$

Formula 3: $\arctan \left(\boldsymbol{\delta}_{\mathbf{m}}\right)^{\mathbf{n}}+\arctan \left(\boldsymbol{\delta}_{\mathbf{m}}\right)^{\mathbf{3 n}}=2 \times \arctan \left(\boldsymbol{\delta}_{\mathbf{k}}\right) ;$ where $\mathbf{k}=\mathbf{2} \times \mathbf{L}(\mathbf{n})$

Formulae $\mathbf{1}$ and $\mathbf{3}$ are applicable for all Metallic Ratios, while Formula $\mathbf{2}$ is applicable only in case of Golden Ratio $\left(\boldsymbol{\delta}_{1}\right.$ or $\left.\boldsymbol{\varphi}\right)$.

Formula 1: For elaboration of Formula (1), consider the Pell Numbers Sequence [4], which is associated with the Second Metallic Mean $\left(\boldsymbol{\delta}_{2}\right)$, that is the Silver Ratio.

\begin{tabular}{|c|l|l|l|l|l|l|l|l|}
\hline $\mathbf{n}=$ & 1 & 2 & 3 & 4 & 5 & 6 & 7 & 8 \\
\hline Pell Numbers & 1 & 2 & 5 & 12 & 29 & 70 & 169 & 408 \\
\hline
\end{tabular}

Now, consider Formula (1):

$$
\arctan \left(\boldsymbol{\delta}_{\mathbf{m}}\right)^{\mathbf{n}}+\arctan \left(\boldsymbol{\delta}_{\mathbf{m}}\right)^{\mathbf{n + 2}}=2 \times \arctan \left(\boldsymbol{\delta}_{\mathbf{k}}\right) ; \text { where } \mathrm{k}=2 \times \mathrm{G}_{(\mathrm{n}+1)}
$$

Let us put the value of Silver Ratio $(\mathbf{1}+\sqrt{\mathbf{2}})$ and $\mathbf{n}$ as any Odd Power of the Silver ratio in this formula.

Putting value of Silver Ratio $\left(\boldsymbol{\delta}_{2}\right)=(\mathbf{1}+\sqrt{\mathbf{2}})=2.4142 \ldots \ldots$ and $\mathbf{n}=\mathbf{3}$ in this formula gives the accurate value of $\mathbf{2 4}^{\text {th }}$ Metallic Ratio. Here, $\mathbf{n = 3}$, hence $\mathbf{k}=$ twice of $(3+1)^{\text {th }}$ Pell Number i.e. $2 \times 12=\mathbf{2 4}$.

$\arctan (\mathbf{1}+\sqrt{\mathbf{2}})^{\mathbf{3}}+\arctan (\mathbf{1}+\sqrt{\mathbf{2}})^{\mathbf{5}}=2 \times \arctan \left(\boldsymbol{\delta}_{\mathbf{2 4}}\right)=2 \times \arctan (24.04159 \ldots \ldots \ldots)$

Similarly, putting $\mathbf{n}=\mathbf{7}$; we get value of $\mathbf{8 1 6}^{\text {th }}$ Metallic Mean. Here, $\mathbf{k}=2 \times(7+1)^{\text {th }}$ Pell Number i.e. $2 \times 408=\mathbf{8 1 6}$.

$\arctan (\mathbf{1}+\sqrt{\mathbf{2}})^{\mathbf{7}}+\arctan (\mathbf{1}+\sqrt{\mathbf{2}})^{\mathbf{9}}=2 \times \arctan \left(\boldsymbol{\delta}_{\mathbf{8 1 6}}\right)=2 \times \arctan (816.001225 \ldots \ldots)$ 
Formula 2: For elaboration of Formula (2) which is applicable only to the Golden Ratio, which is the first of all Metallic Means, consider the Fibonacci Sequence. [3]

\begin{tabular}{|c|c|c|c|c|c|c|c|c|c|c|}
\hline $\mathbf{n}=$ & 1 & 2 & 3 & 4 & 5 & 6 & 7 & 8 & 9 & 10 \\
\hline Fibonacci Numbers & 1 & 1 & 2 & 3 & 5 & 8 & 13 & 21 & 34 & 55 \\
\hline
\end{tabular}

Now consider the formula (2): $\arctan (\boldsymbol{\varphi})^{\mathbf{n}}+\arctan (\boldsymbol{\varphi})^{\mathbf{n}+\mathbf{6}}=2 \times \arctan \left(\boldsymbol{\delta}_{\mathbf{k}}\right) ;$ where $k=F_{(n+3)}$

Putting $\mathbf{n}=\mathbf{3}$, the abovementioned formula gives value of $\mathbf{8}^{\text {th }}$ Metallic Ratio $\left[\mathbf{k}=F_{(n+3)}=F_{6}=\mathbf{8}\right.$ ];

$\arctan (\boldsymbol{\varphi})^{\mathbf{3}}+\arctan (\boldsymbol{\varphi})^{\mathbf{9}}=2 \times \arctan \left(\boldsymbol{\delta}_{8}\right)=2 \times \arctan (8.1231056 \ldots .$.

Similarly, if we put $\mathbf{n}=\mathbf{1}$, the formula gives value of $\mathbf{3}^{\text {rd }}$ Metallic Mean $\left[\mathbf{k}=F_{(n+3)}=F_{4}=\mathbf{3}\right.$ ];

$\arctan (\boldsymbol{\varphi})^{\mathbf{1}}+\arctan (\boldsymbol{\varphi})^{\mathbf{7}}=2 \times \arctan \left(\boldsymbol{\delta}_{3}\right)=2 \times \arctan (3.3027756 \ldots .$.

Formula 3: For elaboration of Formula (3), consider the Integer Sequence associated with Bronze Ratio i.e. the $3^{\text {rd }}$ Metallic Ratio (which may be called as the Bronze Sequence) and the Lucas Numbers associated with these Bronze Numbers.

\begin{tabular}{|l|l|l|l|l|l|l|l|l|l|}
\hline $\mathbf{n}=$ & 1 & 2 & 3 & 4 & 5 & 6 & 7 & 8 & 9 \\
\hline Bronze Numbers & 1 & 3 & 10 & 33 & 109 & 360 & 1189 & 3927 & 12970 \\
\hline Bronze-Lucas Numbers & 3 & 11 & 36 & 119 & 393 & 1298 & 4287 & 14159 & 46764 \\
\hline
\end{tabular}

Consider Formula (3);

$\arctan \left(\boldsymbol{\delta}_{\mathbf{m}}\right)^{\mathbf{n}}+\arctan \left(\boldsymbol{\delta}_{\mathbf{m}}\right)^{\mathbf{3 n}}=2 \times \arctan \left(\boldsymbol{\delta}_{\mathbf{k}}\right) ;$ where $\mathbf{k}=\mathbf{2} \times \mathbf{L}(\mathbf{n})$

Putting value of Bronze Ratio $\left(\boldsymbol{\delta}_{3}\right)=\frac{3+\sqrt{\mathbf{1 3}}}{2}=\mathbf{3 . 3 0 2 7 7 5 6 \ldots . .}$ and $\mathbf{n = 1}$ in this formula gives the accurate value of $\mathbf{6}^{\text {th }}$ Metallic Ratio. Here $\mathbf{n}=\mathbf{1}$, hence $\mathbf{K}=$ twice of $1^{\text {st }}$ Bronze-Lucas Number i.e. $2 \times 3=\mathbf{6}$;

$\arctan (3.3027756 \ldots . .)^{\mathbf{1}}+\arctan (3.3027756 \ldots)^{\mathbf{3}}=2 \times \arctan \left(\boldsymbol{\delta}_{6}\right)=2 \times \arctan (6.16227766 \ldots .$.

Similarly, if we put $\mathbf{n}=\mathbf{3}$; we get the value of $\mathbf{7 2}^{\text {nd }}$ Metallic Mean. Here, $\mathbf{n}=\mathbf{3}$, hence $\mathbf{k}=$ twice of $3^{\text {rd }}$ Bronze-Lucas Number i.e. $2 \times 36=\mathbf{7 2}$;

$\arctan (3.3027756 \ldots .)^{\mathbf{3}}+\arctan (3.3027756 \ldots . .)^{\mathbf{9}}=2 \times \arctan \left(\boldsymbol{\delta}_{72}\right)=2 \times \arctan (72.01388621 \ldots)$ 


\section{The Lucas Sequences and the Odd Powers of different Metallic Ratios:}

Finally, author would like to mention an intriguing fact about the recurrences and interrelationships among the Metallic Ratios. If $\mathbf{L}_{\mathbf{n}}$ is the $\mathbf{n}^{\text {th }}$ term of the Lucas Sequence associated with a Metallic Ratio $\boldsymbol{\delta}_{\mathbf{m}}$, then any odd power of that Metallic Ratio $\left(\boldsymbol{\delta}_{\mathrm{m}}\right)^{\mathbf{n}}$ equals the $\left(\mathrm{L}_{\mathrm{n}}\right)^{\text {th }}$ Metallic Mean.

For example, consider Lucas Sequence associated with the Golden Ratio $(\varphi)$, likewise the Pell-Lucas Sequence associated with Silver Ratio $\left(\boldsymbol{\delta}_{2}\right)$, and the Bronze-Lucas Sequence associated with Bronze Ratio $\left(\boldsymbol{\delta}_{\mathbf{3}}\right)$, as given in the table below. [3],[4]

\begin{tabular}{|l|l|l|l|l|l|l|l|}
\hline \multicolumn{1}{|c|}{$\mathbf{n =}$} & 1 & 2 & 3 & 4 & 5 & 6 & 7 \\
\hline Lucas Numbers : & 1 & 3 & 4 & 7 & 11 & 18 & 29 \\
\hline Pell-Lucas Numbers : & 2 & 6 & 14 & 34 & 82 & 198 & 478 \\
\hline Bronze-Lucas Numbers : & 3 & 11 & 36 & 119 & 393 & 1298 & 4287 \\
\hline
\end{tabular}

\section{Consider any Odd terms of Fibonacci-Lucas Sequence:}

As $\mathrm{L}_{1}=1$, hence $\boldsymbol{\delta}_{\mathrm{L}_{1}}=\boldsymbol{\delta}_{1}=\boldsymbol{\varphi}^{\mathbf{1}}=\mathbf{1}^{\text {st }}$ Metallic Ratio $=1.618 \ldots \ldots$.

As $\mathrm{L}_{3}=4$, hence $\boldsymbol{\delta}_{\mathrm{L}_{3}}=\boldsymbol{\delta}_{4}=\varphi^{3}=4^{\text {th }}$ Metallic Ratio $=4.236 \ldots \ldots$

As $\mathrm{L}_{5}=11$, hence $\boldsymbol{\delta}_{\mathrm{L}_{5}}=\boldsymbol{\delta}_{11}=\boldsymbol{\varphi}^{\mathbf{5}}=\mathbf{1 1}^{\text {th }}$ Metallic Ratio $=11.090 \ldots \ldots$

As $\mathrm{L}_{7}=29$, hence $\boldsymbol{\delta}_{\mathrm{L}_{7}}=\boldsymbol{\delta}_{29}=\boldsymbol{\varphi}^{\mathbf{7}}=\mathbf{2 9}^{\text {th }}$ Metallic Ratio $=29.03444 \ldots \ldots$ and so on.

Similarly, consider any Odd terms of the Pell-Lucas Sequence:

As $\mathrm{PL}_{3}=14$, hence $\boldsymbol{\delta}_{\mathrm{L}_{3}}=\boldsymbol{\delta}_{14}=\left(\boldsymbol{\delta}_{2}\right)^{\mathbf{3}}=\mathbf{1 4}^{\text {th }}$ Metallic Ratio $=14.071 \ldots \ldots$.

As PL $\mathrm{L}_{5}=82$, hence $\boldsymbol{\delta}_{\mathrm{L}_{\mathbf{5}}}=\boldsymbol{\delta}_{\mathbf{8 2}}=\left(\boldsymbol{\delta}_{2}\right)^{\mathbf{5}}=\mathbf{8 2}^{\text {nd }}$ Metallic Ratio $=82.1219 \ldots \ldots .$. and so on .

Likewise, the Odd terms of the Bronze-Lucas Sequence:

As $\mathrm{L}_{3}=36$, hence $\boldsymbol{\delta}_{\mathrm{L}_{3}}=\boldsymbol{\delta}_{36}=\left(\boldsymbol{\delta}_{\mathbf{3}}\right)^{\mathbf{3}}=\mathbf{3 6}^{\text {th }}$ Metallic Ratio $=36.0277 \ldots \ldots$.

As $\mathrm{L}_{5}=393$, hence $\boldsymbol{\delta}_{\mathrm{L}_{5}}=\boldsymbol{\delta}_{393}=\left(\boldsymbol{\delta}_{3}\right)^{\mathbf{5}}=\mathbf{3 9 3 ^ { \text { rd } }}$ Metallic Ratio $=393.0025 \ldots \ldots \ldots$

As $\mathrm{L}_{7}=4287$, hence $\boldsymbol{\delta}_{\mathrm{L}_{7}}=\boldsymbol{\delta}_{4287}=\left(\boldsymbol{\delta}_{3}\right)^{\mathbf{7}}=\mathbf{4 2 8}^{\text {th }}$ Metallic Ratio $=4287.000233 \ldots \ldots$. , etc. 
Hence, to generalise, where $\mathbf{L}_{\mathbf{n}}$ is the $\mathbf{n}^{\text {th }}$ term of the Lucas Sequence associated with the $\mathbf{m}^{\text {th }}$ Metallic Ratio $\left(\boldsymbol{\delta}_{\mathrm{m}}\right)$, and $\mathbf{n}$ is an Odd Positive Integer, the $\mathbf{n}^{\text {th }}$ power of the Metallic Ratio $\boldsymbol{\delta}_{\mathrm{m}}$ equals the $\left(\mathbf{L}_{\mathbf{n}}\right)^{\text {th }}$ Metallic Mean.

$$
\left(\boldsymbol{\delta}_{\mathrm{m}}\right)^{\mathrm{n}}=\boldsymbol{\delta}_{\left(\mathrm{L}_{\mathrm{n}}\right)}
$$

And therefore, interestingly enough, various Metallic Ratios can be expressed in terms of multiple Odd Powers of different Metallic Means, using the trignometric form of the formula mentioned earlier: $\boldsymbol{\delta}_{\mathrm{k}}=\frac{\boldsymbol{\delta}_{\mathrm{m}} \boldsymbol{\delta}_{\mathrm{n}}+\mathbf{1}}{\boldsymbol{\delta}_{\mathrm{m}}-\boldsymbol{\delta}_{\mathrm{n}}}$ : where $\boldsymbol{\delta}_{\mathrm{k}}, \boldsymbol{\delta}_{\mathrm{m}}$ and $\boldsymbol{\delta}_{\mathrm{n}}$ are the $\mathrm{k}^{\mathrm{th}}, \mathrm{m}^{\text {th }}$ and $\mathrm{n}^{\text {th }}$ Metallic Means respectively, and $\mathbf{K}, \mathbf{m}$ and $\mathbf{n}$ are positive integers such that $\mathrm{k}=\frac{\mathrm{mn}+4}{\mathrm{~m}-\mathrm{n}}$

For example, as the $\mathbf{5}^{\text {th }}$ power of Golden Ratio $\boldsymbol{\varphi}^{\mathbf{5}}$ equals the $\mathbf{1 1}^{\text {th }}$ Metallic Ratio; therefore

$$
\begin{aligned}
& \arctan \left(\boldsymbol{\delta}_{12}\right)-\arctan \varphi^{5}=\arctan \left[\frac{1}{\delta_{136}}\right] \\
& \arctan \left(\boldsymbol{\delta}_{16}\right)-\arctan \varphi^{5}=\arctan \left[\frac{1}{\delta_{36}}\right] \\
& \arctan \left(\boldsymbol{\delta}_{36}\right)-\arctan \varphi^{5}=\arctan \left[\frac{1}{\delta_{16}}\right] \\
& \arctan \left(\boldsymbol{\delta}_{136}\right)-\arctan \varphi^{5}=\arctan \left[\frac{1}{\delta_{12}}\right]
\end{aligned}
$$

Similarly, as the cube of Silver Ratio $\left(\boldsymbol{\delta}_{2}\right)^{\mathbf{3}}$ equals the $\mathbf{1 4}^{\text {th }}$ Metallic Ratio; and hence

$$
\begin{aligned}
& \arctan \left(\boldsymbol{\delta}_{214}\right)-\arctan \left(\boldsymbol{\delta}_{2}\right)^{\mathbf{3}}=\arctan \left[\frac{1}{\delta_{15}}\right] \\
& \arctan \left(\boldsymbol{\delta}_{114}\right)-\arctan \left(\boldsymbol{\delta}_{2}\right)^{\mathbf{3}}=\arctan \left[\frac{1}{\delta_{16}}\right] \\
& \arctan \left(\boldsymbol{\delta}_{64}\right)-\arctan \left(\boldsymbol{\delta}_{2}\right)^{\mathbf{3}}=\arctan \left[\frac{1}{\delta_{18}}\right] \\
& \arctan \left(\boldsymbol{\delta}_{54}\right)-\arctan \left(\boldsymbol{\delta}_{2}\right)^{\mathbf{3}}=\arctan \left[\frac{1}{\delta_{19}}\right] \\
& \arctan \left(\boldsymbol{\delta}_{39}\right)-\arctan \left(\boldsymbol{\delta}_{2}\right)^{\mathbf{3}}=\arctan \left[\frac{1}{\delta_{22}}\right]
\end{aligned}
$$




$$
\begin{aligned}
& \arctan \left(\boldsymbol{\delta}_{34}\right)-\arctan \left(\boldsymbol{\delta}_{2}\right)^{\mathbf{3}}=\arctan \left[\frac{1}{\delta_{24}}\right] \\
& \arctan \left(\boldsymbol{\delta}_{24}\right)-\arctan \left(\boldsymbol{\delta}_{2}\right)^{\mathbf{3}}=\arctan \left[\frac{1}{\delta_{34}}\right] \\
& \arctan \left(\boldsymbol{\delta}_{22}\right)-\arctan \left(\boldsymbol{\delta}_{2}\right)^{\mathbf{3}}=\arctan \left[\frac{1}{\delta_{39}}\right] \\
& \arctan \left(\boldsymbol{\delta}_{19}\right)-\arctan \left(\boldsymbol{\delta}_{2}\right)^{\mathbf{3}}=\arctan \left[\frac{1}{\delta_{54}}\right] \\
& \arctan \left(\boldsymbol{\delta}_{18}\right)-\arctan \left(\boldsymbol{\delta}_{2}\right)^{\mathbf{3}}=\arctan \left[\frac{1}{\delta_{64}}\right] \\
& \arctan \left(\boldsymbol{\delta}_{16}\right)-\arctan \left(\boldsymbol{\delta}_{2}\right)^{\mathbf{3}}=\arctan \left[\frac{1}{\delta_{114}}\right] \\
& \arctan \left(\boldsymbol{\delta}_{15}\right)-\arctan \left(\boldsymbol{\delta}_{2}\right)^{\mathbf{3}}=\arctan \left[\frac{1}{\delta_{214}}\right]
\end{aligned}
$$

Similar expressions can be derived for various Metallic Means, with the multiple Odd Powers of different Metallic Ratios.

\section{Conclusion:}

This paper put forward certain peculiar formulae those provide the precise mathematical relationships between different Metallic Ratios.

To summarise, where $\mathrm{K}, \mathrm{m}$ and $\mathrm{n}$ being the positive integers such that; $\frac{\mathrm{mn}+4}{\mathrm{~m}-\mathrm{n}}=\mathrm{k}$; and $\boldsymbol{\delta}_{\mathrm{k}} \boldsymbol{\delta}_{\mathrm{m}}$ and $\boldsymbol{\delta}_{\mathrm{n}}$ are the $\mathrm{k}^{\text {th }}, \mathrm{m}^{\text {th }}$ and $\mathrm{n}^{\text {th }}$ Metallic Means respectively, then $\boldsymbol{\delta}_{\mathrm{k}}=\frac{\delta_{\mathrm{m}} \times \delta_{\mathrm{n}}+1}{\delta_{\mathrm{m}}-\delta_{\mathrm{n}}}$

Moreover, various "Odd Powers" of a Metallic Ratio are found to render the precise values of several Metallic Means by the following three formulae;

$\arctan \left(\boldsymbol{\delta}_{\mathbf{m}}\right)^{\mathbf{n}}+\arctan \left(\boldsymbol{\delta}_{\mathbf{m}}\right)^{\mathbf{n}+\mathbf{2}}=2 \times \arctan \left(\boldsymbol{\delta}_{\mathbf{k}}\right) ;$ where $\mathrm{k}=2 \times \mathrm{G}_{(\mathrm{n}+1)}$

$\arctan (\boldsymbol{\varphi})^{\mathbf{n}}+\arctan (\boldsymbol{\varphi})^{\mathbf{n}+\mathbf{6}}=2 \times \arctan \left(\boldsymbol{\delta}_{\mathbf{k}}\right) ;$ where $\mathrm{k}=\mathrm{F}_{(\mathrm{n}+3)}$ : applicable only for Golden Ratio; $\arctan \left(\boldsymbol{\delta}_{\mathbf{m}}\right)^{\mathbf{n}}+\arctan \left(\boldsymbol{\delta}_{\mathbf{m}}\right)^{\mathbf{3 n}}=2 \times \arctan \left(\boldsymbol{\delta}_{\mathbf{k}}\right) ;$ where $\mathrm{k}=2 \times \mathrm{L}(\mathrm{n})$

Also, where $\mathbf{L}_{\mathbf{n}}$ is the $\mathbf{n}^{\text {th }}$ Lucas term associated with the Metallic Ratio $\left(\boldsymbol{\delta}_{\mathbf{m}}\right)$, and $\mathbf{n}$ is an Odd Positive Integer, then $\mathbf{n}^{\text {th }}$ power of the Metallic Ratio $\boldsymbol{\delta}_{\mathrm{m}}$ equals the $\left(\mathbf{L}_{\mathbf{n}}\right)^{\text {th }}$ Metallic Mean, that is $\left(\boldsymbol{\delta}_{\mathrm{m}}\right)^{\mathbf{n}}=\boldsymbol{\delta}_{\left(\mathrm{L}_{\mathrm{n}}\right)}$. And, this enables us to express a Metallic Mean in terms of the various Odd Powers of different Metallic Ratios. 
To conclude, different Metallic Means are found to be closely related with each other, and the precise correlations are given by the mathematical formulae communicated in this paper.

\section{References:}

[1] Rajput, Chetansing. (2021). Golden Ratio. JOURNAL OF ADVANCES IN MATHEMATICS. 20. 19-42. https://doi.org/10.24297/jam.v20i.8945

[2] Rajput, Chetansing. (2021). Metallic Means and Right Triangles: The Geometric Substantiation of all Metallic Ratios. JOURNAL OF ADVANCES IN MATHEMATICS. 20. 167-173. https://doi.org/10.24297/jam.v20i.9029 and https://doi.org/10.24297/jam.v20i.9034

[3] Sloane, N. J. A. Sequences A000045/M0692, A000204/M2341 in "The On-Line Encyclopedia of Integer Sequences."

https://oeis.org/A000045 and https://oeis.org/A000204

[4] Sloane, N. J.A. Sequences A000129/M1413, A002203/M0360, in "The On-Line Encyclopedia of Integer Sequences."

http://oeis.org/A000129 and http://oeis.org/A002203

Conflict of Interest: There is no Conflict of Interest.

Funding Statement: The research is self-sponsored by the author. 